\title{
3D-Fractal Engineering Based on Oxide-only Corner Lithography
}

\author{
J.W. Berenschot, R.M. Tiggelaar*, J. Geerlings, \\ J.G.E. Gardeniers, N.R. Tas \\ Mesoscale Chemical Systems ( ${ }^{*}$ NanoLab) \\ $\mathrm{MESA}^{+}$Institute for Nanotechnology \\ University of Twente \\ Enschede, The Netherlands \\ n.r.tas@utwente.nl
}

\author{
M. Malankowska, M.P. Pina, R. Mallada \\ Chemical and Environmental Engineering \\ Aragon Nanoscience institute (INA) \\ University of Zaragoza \\ Zaragoza, Spain
}

\begin{abstract}
This paper reports a new highly simplified machining process for three dimensional (3D)-fractal nanofabrication based on oxide-only corner lithography. It consists of a repeated sequence of wet etching (silicon), thermal oxidation and wet etching (silicon oxide). The previously reported 3D-fractal fabrication process needed additional low pressure chemical vapor deposition (LPCVD) steps of silicon nitride, as well as local oxidation of silicon (LOCOS). Employing this new procedure, a three generation folded silicon oxide fractal sheet with approx. a $10 \mu \mathrm{m}$ footprint has been fabricated.
\end{abstract}

Keywords-nanofabrication, fractals, corner lithography, silicon dioxide, wet etching, single crystalline silicon

\section{INTRODUCTION}

3D engineered fractal structures [1] can find application in numerous fields, like optical sensing, photovoltaics, microfluidic mixing, sieving and catalytic conversion. Previously, a process based on a combination of silicon nitride "corner lithography", anisotropic and isotropic etching, and local oxidation of silicon (LOCOS) was developed to microfabricate 3D fractal structures [1]. This process has now been greatly simplified, and it will be shown in this paper that $3 \mathrm{D}$ fractal structures can be fabricated by a repeated sequence of anisotropic etching, thermal oxidation and isotropic etching only. By means of this oxide-only process various generations of $3 \mathrm{D}$ fractal structures can be made, as schematically shown in Fig. 1.
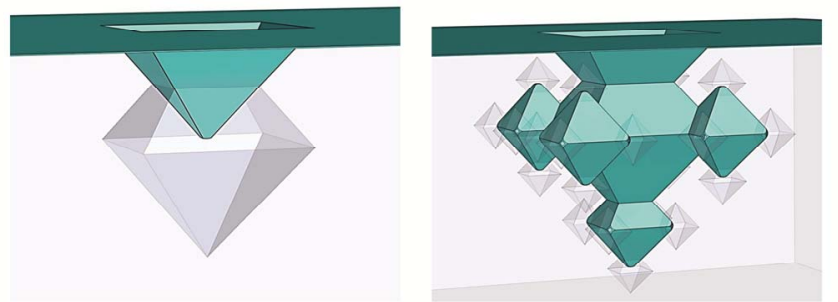

Fig. 1. Schematic representation of $1^{\text {st }}$ (left) and $3^{\text {rd }}$ (right) level 3D fractals

\section{FUNDAMENTALS OF OXIDE-ONLY 3D-FRACTAL ENGINEERING}

Our "conventional" way of engineering of 3D fractal structures, as reported previously, is based on a combination of anistropic etching of silicon and corner lithography [1-3]. In more detail, this conventional method is an iterative process that involves conformal deposition of layers of silicon nitride by LPCVD, and thermal oxide $\left(\mathrm{SiO}_{2}\right)$ in anisotropically etched silicon structures - i.e. inverted pyramids and octahedrons - in combination with selective time-stopped (an)isotropic wet etching. The employed wet etchants to realize $3 \mathrm{D}$ fractals are potassium hydroxide $(\mathrm{KOH})$ and tetra methyl ammonium hydroxide (TMAH) to shape (100)-oriented single crystalline silicon, and (buffered) hydrofluoric acid ((B)HF) and phosphoric acid $\left(\mathrm{H}_{3} \mathrm{PO}_{4}\right)$ to isotropically and selectively pattern conformal films of silicon nitride and $\mathrm{SiO}_{2}$. Although this conventional method yields large arrays of 3D fractal structures, in practice it is a time-consuming and rather expensive procedure, hence the need for a much more simplified process flow. We have found such a procedure, based on the notion that silicon nitride based corner lithography in combination with LOCOS, can be replaced by a single silicon oxidation step, making use of the stress induced thinning of silicon oxide in sharp concave corners [4].

In order to be able to fabricate 3D fractals with oxide-only corner lithography, the grown oxide layer should be conformal on convex corners as well as equally thick on the silicon (100) and (111) crystal planes. If these requirements are not fulfilled the layer of $\mathrm{SiO}_{2}$ cannot be properly patterned by means of time-stopped isotropic etching (i.e. due to thickness variations the $\mathrm{SiO}_{2}$ is removed from locations where it should remain), or will not function as a proper mask during selective anisotropic etching of silicon. Therefore, this simplified process uses (dry) thermal oxidation at $1100^{\circ} \mathrm{C}$. Oxidation of silicon at this temperature leads to fundamental differences in the grown oxide compared to thermal oxidation at relatively low temperatures $\left(\leq 950^{\circ} \mathrm{C}\right)$, in terms of layer thickness on $(100)$ and (111)-silicon crystal planes as well as layer conformality around convex corners. 
At low thermal oxidation temperatures $\left(\leq 950^{\circ} \mathrm{C}\right)$ the oxide thickness at convex and concave corners is thinner than a flat (100)-Si planes due to compressive stress at the corner structures [5],[6]. At temperatures of $1000{ }^{\circ} \mathrm{C}$ the formed oxide layer on convex corners is not thinned with respect to the layer thickness on planar (100)-Si, but at this temperature there is a difference in oxide growth rate on the main crystal directions of silicon [7]. Upon dry thermal oxidation of silicon at $1100{ }^{\circ} \mathrm{C}$ the mentioned aspects regarding non-conformality on convex corners and differences in oxide layer thickness on (100) and (111) Si-planes are avoided [7]. In concave corners the severe compressive stress that develops [8] does not relief, and the connected reduction in oxidation rate leads to a locally thinner layer.

The degree of sharpening of the thermal oxide layer in concave corners depends on the amount of intersecting (111)- planes: the higher the number of intersecting planes, the thinner the grown oxide layer. Thus, in ribbons - i.e. two intersecting (111)-planes - less oxide sharpening occurs compared to an intersection of three or four (111)-planes (i.e. apices) (Fig.2). These aspects yield the possibility to solely remove the $\mathrm{SiO}_{2}$ from apices by means of timed isotropic etching in $1 \% \mathrm{HF}$, while oxide remains in ribbons and on planes. This is illustrated in Fig. 2.

The procedure to self-form the 3D-fractal now becomes very simple: after thermal oxidation and timed-HF etching, at each apex the underlying $\mathrm{Si}$ can be selectively etched (anisotropic etching in TMAH), resulting in the formation of a next level octahedral structures at all apices simultaneously. Repetition of this simple sequence of anisotropic Si-etching / thermal oxidation at $1100^{\circ} \mathrm{C} /$ isotropic $\mathrm{SiO}_{2}$-etching results in multi-level 3D-fractal structures.

\section{apex}

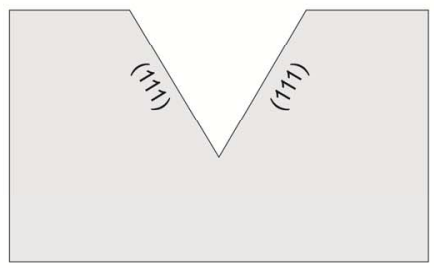

a) Anisotropic etching and mask stripping

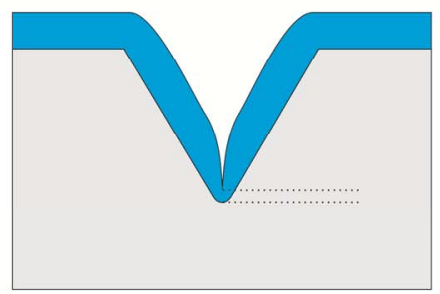

b) Oxidation

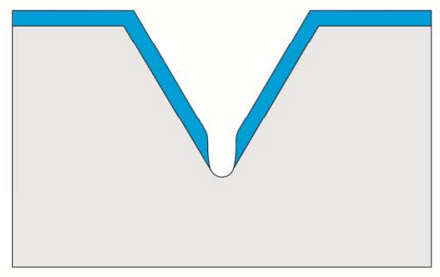

c) Isotropic etching

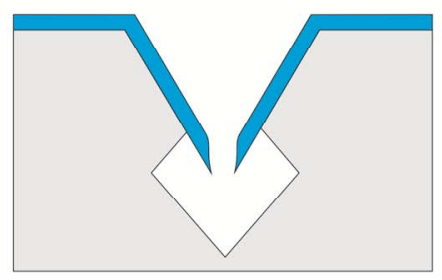

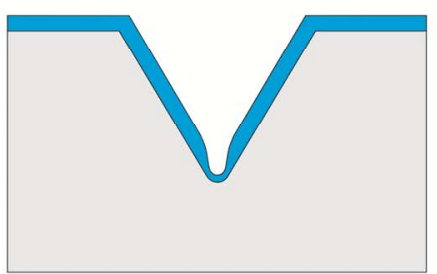

\section{2 planes}
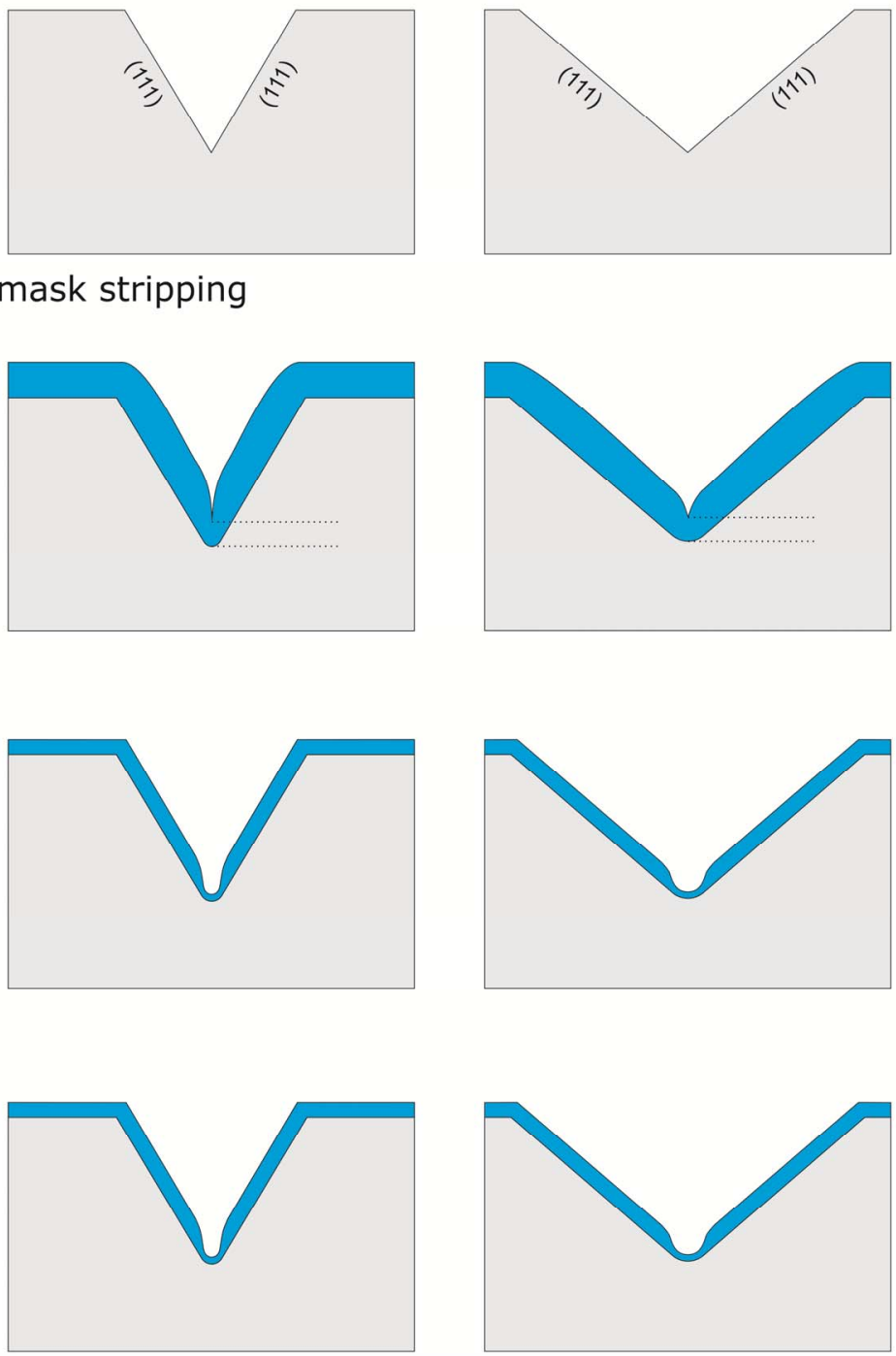

\section{d) Anisotropic etching}

Fig. 2. Selective opening of the thermally grown silicon oxide at the apex of the pyramidal pit after HF etching. Note that stress induced oxidation retardation is more pronounced in concave corners where more than two planes intersect. 

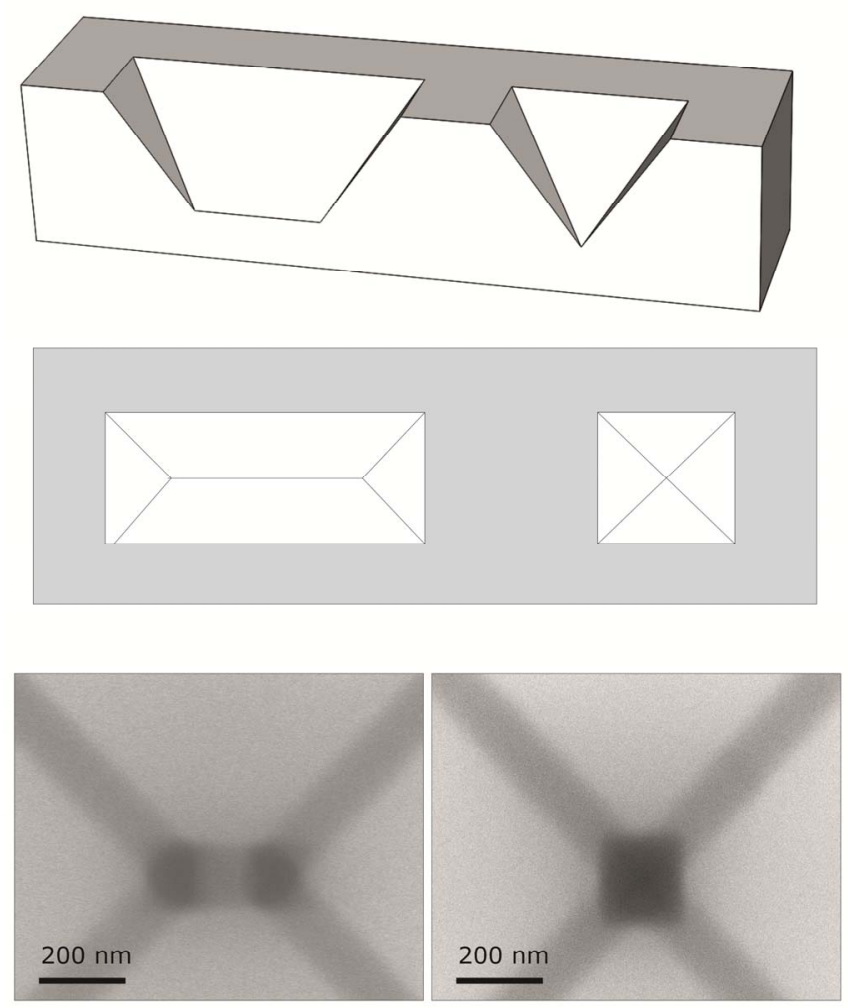

Fig. 3. Top and middle: 3D and topview schematic representations of 2, 3 and 4 intersecting (111)-Si planes. Bottom: topview SEM-images of insections of 2, 3 and 4 (111)-Si planes upon etching in HF: timedependent opening of the apices is visible.

\section{EXPERIMENTAL RESULTS AND DISCUSSION}

To illustrate the selective opening of apices, we etched an inverted pyramid in (100)-Si using $\mathrm{KOH}\left(25 \mathrm{wt} . \%, 70{ }^{\circ} \mathrm{C}\right)$, with a slightly rectangular (Fig. 3, left), and square (Fig. 3, right) footprint. These structures were subsequently oxidized (dry, $1100^{\circ} \mathrm{C}$ for $95 \mathrm{~min}$ ), resulting in a $\mathrm{SiO}_{2}$ thickness of 160

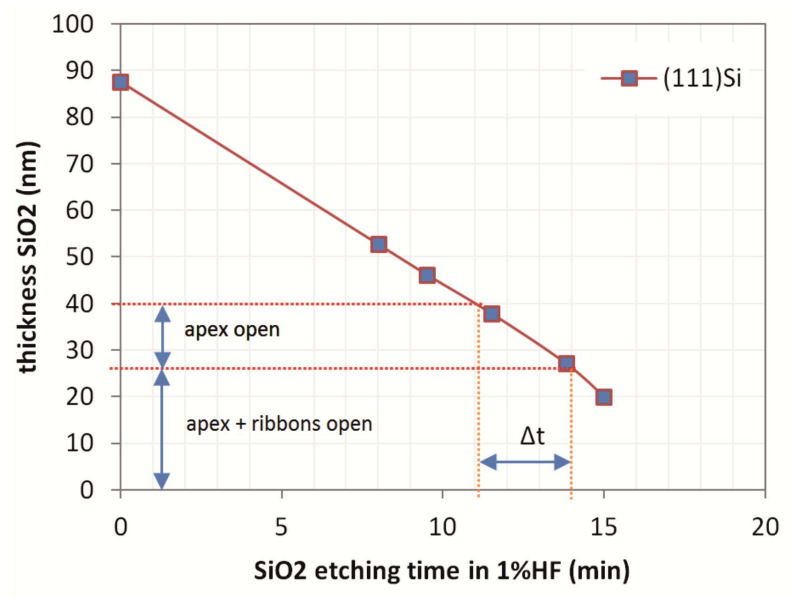

$\mathrm{nm}$ and $155 \mathrm{~nm}$ on (111) and (100) oriented surfaces, respectively. Fig. 3 shows SEM images (top view) after $19 \mathrm{~min}$ $+30 \mathrm{sec}$ etching in $1 \% \mathrm{HF}$ (etch rate $4.4 \pm 0.1 \mathrm{~nm} / \mathrm{min}$ ) and 5 min of TMAH etching $\left(25 \mathrm{wt} \%, 70{ }^{\circ} \mathrm{C}\right)$ to make a possible opening more visible in the SEM. The remaining oxide thickness on (111) surfaces is $74 \mathrm{~nm}$.

A first indication of the time window $(\Delta t)$ available between opening of only the apices $v s$. opening of the ribbons and apices is given in Fig. 4, for a starting oxide thickness of $88 \mathrm{~nm}$ and $160 \mathrm{~nm}$, respectively (on (111) surfaces). For each measurement point in the graphs, the samples were taken from the $1 \%$ HF solution, etched in TMAH and then inspected by SEM. This sequence was repeated and the opening of apices or ribbons as detected is indicated in the graphs. Note that the indicated time window has a considerable error margin due to the limited number of measurement points.

Starting point for the realization of $3 \mathrm{D}$ fractal structures in an inverted pyramid etched in (100)-Si with $\mathrm{KOH}$, with a square footprint of $5 \mu \mathrm{m}$. After growing a thermal oxide layer with a known thickness (ca. $160 \mathrm{~nm}, 1 \mathrm{~h} 35 \mathrm{~min}$ at $1100{ }^{\circ} \mathrm{C}$ ), a time window exists for which only the apices are free of oxide. For the engineering of 3D fractal structures solely based on oxide corner-lithography, an etch-time of $20 \mathrm{~min} 30 \mathrm{sec}$ in $1 \%$ HF is applied. Post to this HF-step, through the apex, silicon can be etched anisotropically in TMAH $\left(25\right.$ wt. $\left.\%, 70{ }^{\circ} \mathrm{C}\right)$, yielding a new octahedron that is bound by the slow etching (111) Si-planes. For each fabrication level of a fractal structure, the oxidation and isotropic etch time are constant, however, the time-length of the TMAH etch step is halved for each new level (starting with an etch time of $145 \mathrm{~min}$ at level zero). Upon a 3 times repetition of this sequence - TMAH-etching, $1100^{\circ} \mathrm{C}$-oxidation and $\mathrm{SiO}_{2}$-etching - followed by a final thermal oxidation run, anodic bonding with a Mempax glass wafer at $400{ }^{\circ} \mathrm{C}$, and removal of the bulk-Si, freestanding three-generation silicon oxide fractal sheets can be fabricated (Fig. 5). Note that depending on the final step, apices can remain closed or be opened.

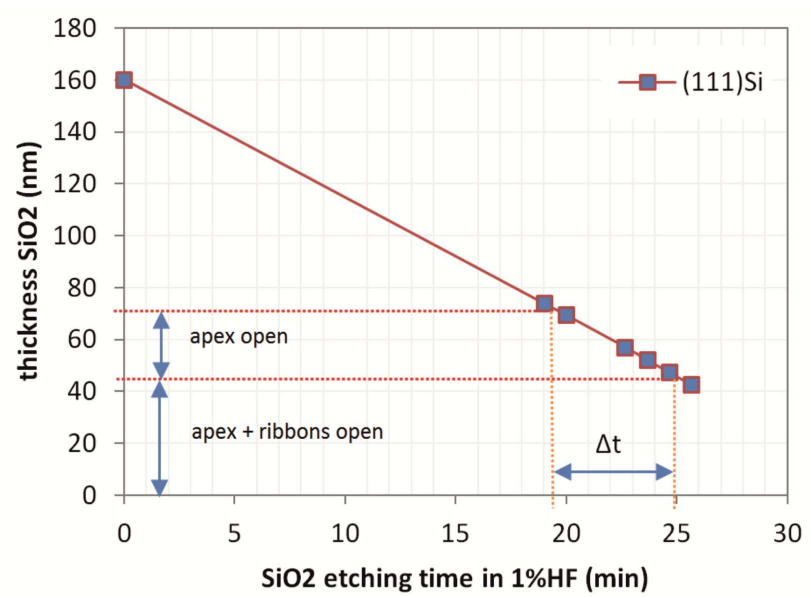

Fig. 4. Remaining oxide thickness in apices and ribbons as function of etching time in $1 \% \mathrm{HF}$ (starting oxide thickness $160 \mathrm{~nm}$ (left) or $88 \mathrm{~nm}$ (right)): within the time window $\Delta \mathrm{t}$ only the apices are opened. 

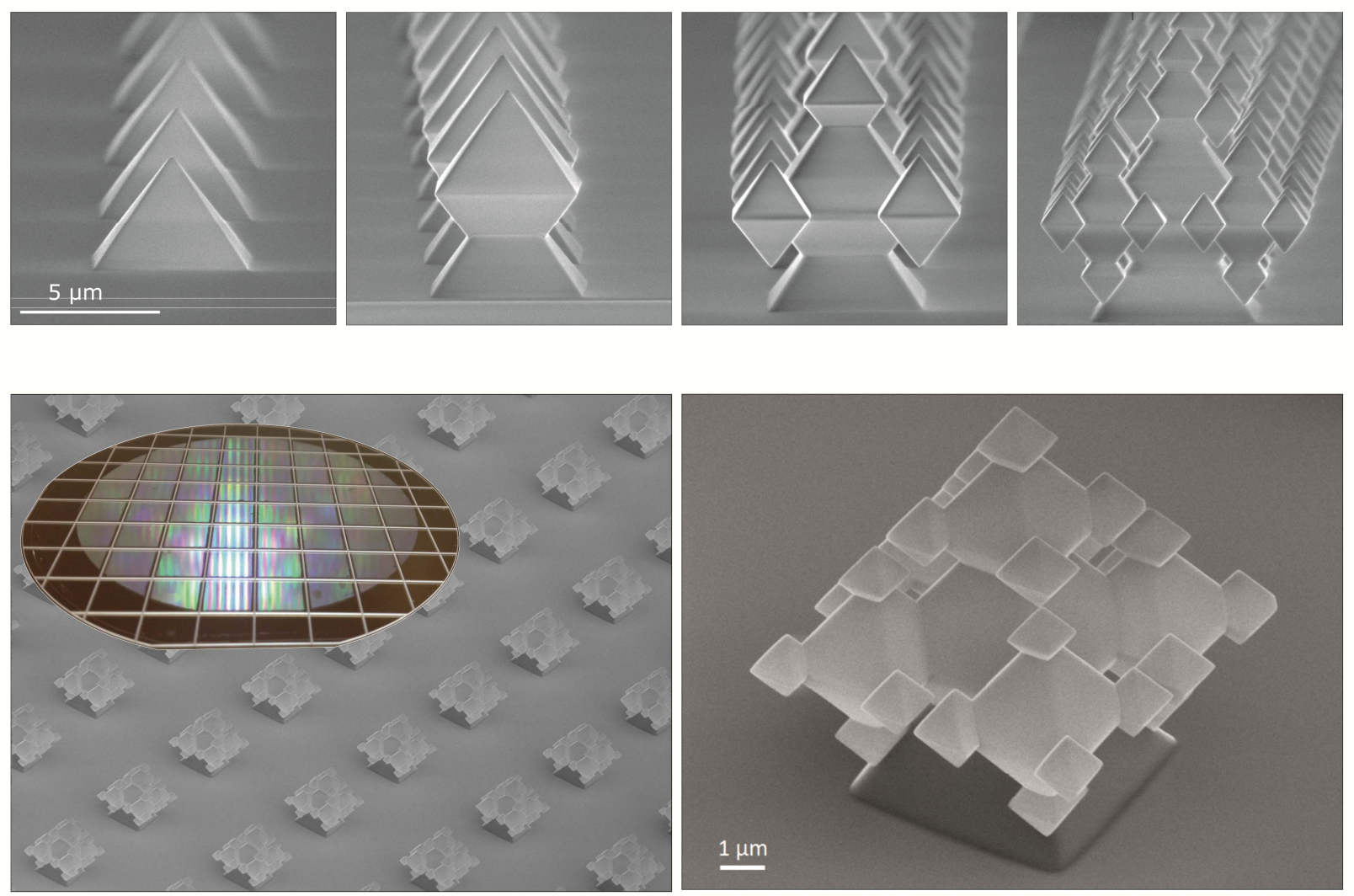

Fig. 3. Top row (left-to-right): SEM images of $0^{\text {th }}, 1^{\text {st }}, 2^{\text {nd }}$ and $3^{\text {rd }}$ generation silicon oxide fractal sheets. Bottom row: overview of fractals on glass substrate (inset: glass wafer with $1 \times 1 \mathrm{~cm}^{2}$ specimen with fractals) and zoom-in on one freestandig $3^{\text {rd }}$ level 3D silicon oxide fractal sheet.

\section{CONCLUSIONS AND OUTLOOK}

We have found a regime in thermal oxidation of silicon, in which the oxide formed is significantly thinner in concave apices, with respect to concave ribbons as well as all convex corners. Based on this regime, it is possible to fabricate $3 \mathrm{D}$ fractals by a combination of anisotropic etching of silicon, thermal oxidation and isotropic HF etching only. This procedure was successfully applied to produce a three generation octahedral fractal structure, with a total size of about $10 \mu \mathrm{m}$ and a smallest sub-structure size of about $1 \mu \mathrm{m}$.

We are currently exploring microfluidic and optical applications of the nanofabricated 3D-fractals.

\section{ACKNOWLEDGMENT}

We acknowledge the generous support of the University of Twente for this explorative research, as well as financial supporting of this work via doctoral research fellowship awarded to Magdalena Malankowska by the European Commission - Education, Audiovisual and Culture Executive Agency (EACEA), under the program: Erasmus Mundus Doctorate in Membrane Engineering - EUDIME (FPA $\mathrm{N}^{\circ}$ 2011-0014).

\section{REFERENCES}

[1] E.J.W Berenschot, H.V. Jansen, and N.R. Tas, "Fabrication of 3D fractal structures using nanoscale anistropic etching of single crystalline silicon," J. Micromech. Microeng., vol. 23, pp. 055024 (10 pp.), 2013.

[2] N. Burouni et al., "Wafer-scale fabrication of nanoapertures using corner lithography,"Nanotechnology, vol. 24, pp. 285303 (10 pp.), 2013.

[3] E.J.W. Berenschot et al., "3D nanofabrication of fluidic components by corner lithography", Small, vol. 8 (24), pp. 3823-3831, 2012.

[4] R. B. Marcus and T. T. Sheng, "The oxidation of shaped silicon surfaces," J. Electrochem. Soc., vol. 129 (6), pp. 1278-1282, 1982

[5] P.N. Minh, T. Ono, and M. Esashi, "Nonuniform silicon oxidation and application for the fabrication of aperture for near-field scanning optical microscopy,” Appl. Phys. Lett., vol. 75 (26), pp. 4076-4078, 1999.

[6] C. Mihalcea, A. Vollkopf, and E. Oesterschulze, "Reproducible largearea microfabrication of sub-100 nm apertures of hollow tips," J. Electrochem. Soc., vol. 147 (5), pp. 1970-1972, 2000.

[7] B.E. Deal, A.S. Grove, "General relationship for the thermal oxidation of silicon," J. Appl. Phys., vol. 36, p. 3770, 1965.

[8] A. Vollkopf, O. Rudow, M. Müller-Wiegand, G. Georgiev, and E. Oesterschulze, "Influence of the oxidation temperature on the fabrication process of silicon dioxide aperture tips," Appl. Phys. A, vol. 76, pp. 923926, 2003. 